\title{
Calcineurin and Its Regulator Sra/DSCR1 Are Essential for Sleep in Drosophila
}

\author{
Yasuhiro Nakai, ${ }^{1 \star}$ Junjiro Horiuchi, ${ }^{1,2 \star}$ Manabu Tsuda, ${ }^{1}$ Satomi Takeo, ${ }^{3}$ Shin Akahori, ${ }^{1}$ Takashi Matsuo, ${ }^{1}$ \\ Kazuhiko Kume, ${ }^{4}$ and Toshiro Aigaki ${ }^{1,2}$ \\ ${ }^{1}$ Department of Biological Sciences, and ${ }^{2}$ Center for Priority Areas, Tokyo Metropolitan University, Hachioji-shi, Tokyo 192-0397, Japan, ${ }^{3}$ Stowers Institute \\ for Medical Research, Kansas City, Missouri 64110, and ${ }^{4}$ Institute of Molecular Embryology and Genetics, Kumamoto University, 860-0811 Kumamoto, \\ Japan
}

Sleep is a fundamental biological process for all animals. However, the molecular mechanisms that regulate sleep are still poorly understood. Here we report that sleep-like behavior in Drosophila is severely impaired by mutations in sarah (sra), a member of the Regulator of Calcineurin (RCAN) family of genes. Sleep reduction in sra mutants is highly correlated with decreases in Sra protein levels. Pan-neural expression of sra rescues this behavioral phenotype, indicating that neuronal sra function is required for normal sleep. Since Sra regulates calcineurin $(\mathrm{CN})$, we generated and examined the behavior of knock-out mutants for all Drosophila CN genes: CanA-14F, $P p 2 B-14 D$, and CanA1 (catalytic subunits), and $C a n B$ and $C a n B 2$ (regulatory subunits). While all mutants show at least minor changes in sleep, CanA$14 F^{K O}$ and $C a n B^{K O}$ have striking reductions, suggesting that these are the major $\mathrm{CN}$ subunits regulating sleep. In addition, neuronal expression of constitutively active forms of $\mathrm{CN}$ catalytic subunits also significantly reduces sleep, demonstrating that both increases and decreases in $\mathrm{CN}$ activity inhibit sleep. sra sleep defects are suppressed by $\mathrm{CN}$ mutations, indicating that sra and CN affect sleep through a common mechanism. Our results demonstrate that $\mathrm{CN}$ and its regulation by Sra are required for normal sleep in Drosophila and identify a critical role of $\mathrm{Ca}^{2+} /$ calmodulin-dependent signaling in sleep regulation.

\section{Introduction}

Although sleep or sleep-like states have been observed in most animal species studied to date, the basic role of sleep is still not well understood. Previous studies identifying sleep mutants have shown that changes in sleep are associated with changes in neuronal activity. In Drosophila, mutations that decrease activity of the shaker voltage-gated $\mathrm{K}^{+}$channel increase neuronal activity and decrease sleep (Cirelli et al., 2005). In addition, activity of various neurotransmitters and neuropeptides, including octopamine (Crocker and Sehgal, 2008), dopamine (Kume et al., 2005), and pigment-dispersing factor (pdf) (Parisky et al., 2008; Chung et al., 2009), are associated with decreased sleep in flies, while activity of others, including GABA (Agosto et al., 2008; Parisky et al., 2008) and serotonin (Yuan et al., 2006), are associated with increased sleep. However, intracellular molecular components mediating the effects of these molecules on sleep have not readily been

Received March 16, 2011; revised June 22, 2011; accepted July 20, 2011.

Author contributions: Y.N., J.H., and T.A. designed research; Y.N., J.H., and S.A. performed research; Y.N., M.T., S.T., T.M., and K.K. contributed unpublished reagents/analytic tools; Y.N., J.H., M.T., S.T., S.A., K.K., and T.A. analyzed data; J.H. and T.A. wrote the paper.

*Y.N. and J.H. contributed equally to this work.

This work was supported by a Grant-in-Aid for Scientific Research (Kakenhi) from the Ministry of Education, Culture, Sports, Science and Technology of Japan to T.A. We thank Minoru Saitoe and Kensuke Horiuchi for critical reading of the manuscript and the Drosophila Genetic Resource Center, Kyoto and the Bloomington Stock Center for providing flies.

Correspondence should be addressed to Toshiro Aigaki, Laboratory of Cellular Genetics, Tokyo Metropolitan University, Minami-osawa, Hachoji, Tokyo 192-0397, Japan. E-mail: aigaki-toshiro@tmu.ac.jp.

DOI:10.1523/JNEUROSCI.1337-11.2011

Copyright $\odot 2011$ the authors $\quad 0270-6474 / 11 / 3112759-08 \$ 15.00 / 0$ identified. $\mathrm{Ca}^{2+} /$ calmodulin $(\mathrm{CaM})$ signaling is known to be critical for synaptic activity since it regulates activity of $\mathrm{Ca}^{2+} /$ CaM-dependent protein kinases and phosphatases essential for plasticity, but a role of $\mathrm{Ca}^{2+} / \mathrm{CaM}$ signaling in sleep has not previously been described.

Calcineurin $(\mathrm{CN})$ is a highly conserved $\mathrm{Ca}^{2+} / \mathrm{CaM}$-dependent protein phosphatase, implicated in synaptic plasticity in mammals (Rusnak and Mertz, 2000). CN is composed of a heterodimer of an $\sim 60 \mathrm{kDa}$ catalytic subunit $(\mathrm{CnA})$ and an $\sim 19 \mathrm{kDa} \mathrm{EF}-$ hand $\mathrm{Ca}^{2+}$-binding regulatory subunit $(\mathrm{CnB})$. Both subunits are essential for phosphatase activity. Besides $\mathrm{Ca}^{2+}$ and $\mathrm{CaM}$, $\mathrm{CN}$ activity also depends on the regulator of $\mathrm{CN}$ (RCAN) family of proteins (Hilioti and Cunningham, 2003; Davies et al., 2007). RCAN proteins were initially identified as $\mathrm{CN}$ inhibitors that bind directly to $\mathrm{CnA}$ subunits to inhibit activity. However, RCAN knock-outs in fungi, Drosophila, and mice exhibit phenotypes similar to $\mathrm{CN}$ mutants, indicating that these proteins function both as $\mathrm{CN}$ activators and inhibitors (Görlach et al., 2000; Vega et al., 2003; Mehta et al., 2009). In Drosophila, the sra gene has been shown to encode the only known RCAN protein as assayed by homology searches, biochemical interactions, and functional genetic interactions (Ejima et al., 2004; Horner et al., 2006; Takeo et al., 2006).

Here we show that both sra mutants and specific $\mathrm{CN}$ subunit gene mutants have severe sleep defects, indicating that $\mathrm{Ca}^{2+}$ / CaM signaling plays a critical role in sleep regulation. These results suggest a mechanistic connection linking synaptic plasticity and sleep. 
A
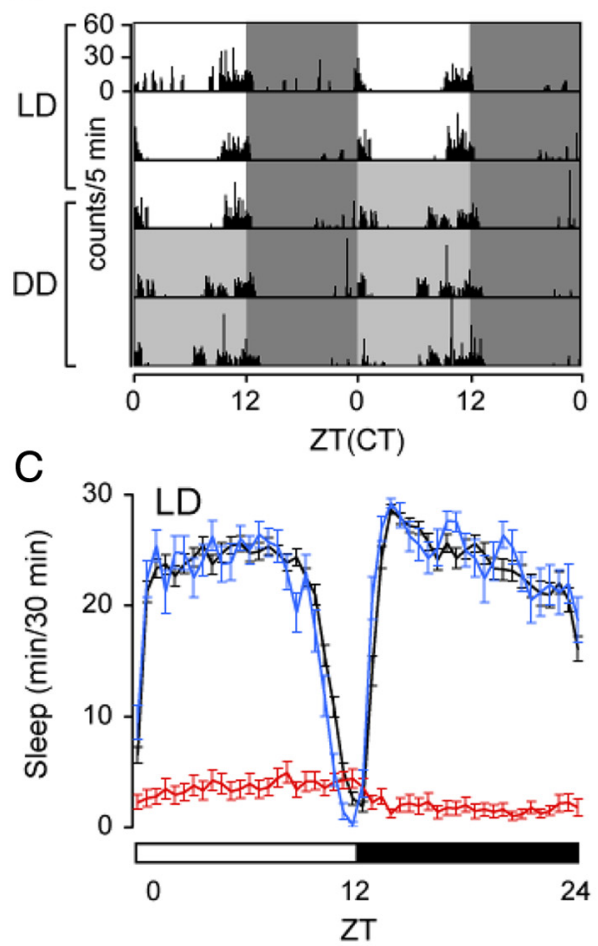

E

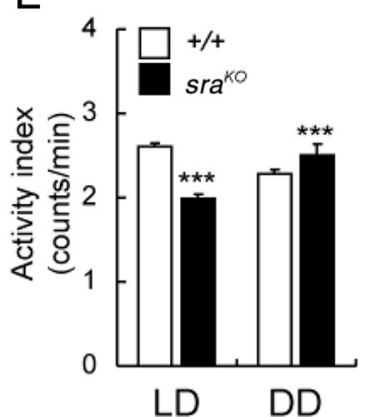

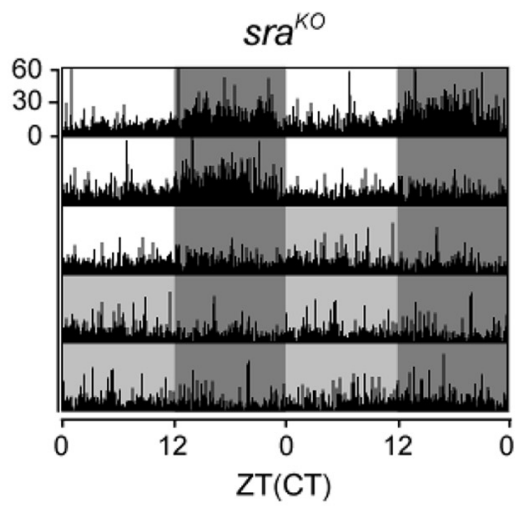

B
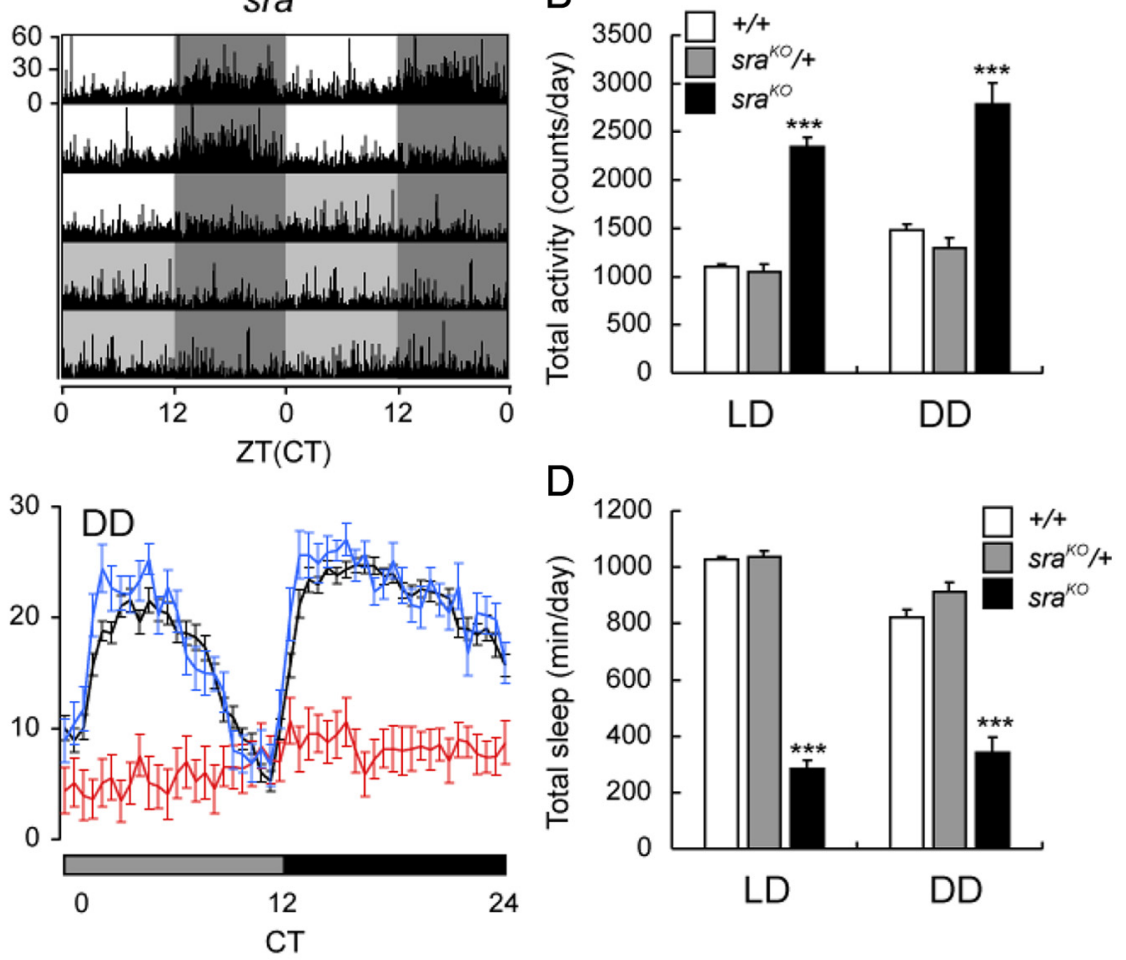

$\mathrm{F}$

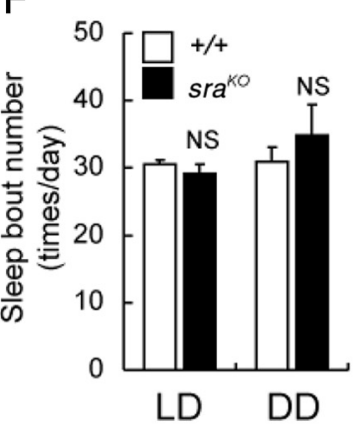

G

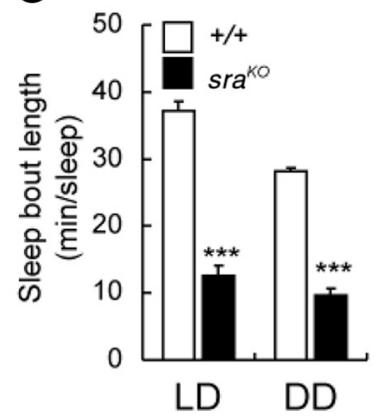

Figure 1. $\quad s r a{ }^{K O}$ mutants exhibit increased locomotor activity and decreased sleep. $A$, Locomotor activity in representative wild-type $(+/+)$ and $s r a{ }^{K O}$ flies in LD and DD conditions $(3 \mathrm{~d}$ each in $L D$ and $D D$ conditions) are double plotted, such that each horizontal line corresponds to $2 \mathrm{~d}$. Data are plotted as number of activity counts (beam crossings) per 5 min. $B$, Total daily activity of $+/+$, $\mathrm{sra}^{K O} /+$, and $s \mathrm{a}^{K O}$ flies plotted in LD and DD conditions. C, Daily sleep profiles of $+/+$ (black), $s r a^{K O} /+$ (blue), and $s r a{ }^{K O}$ (red) flies in LD and DD conditions. The amount of sleep per 30 min bin is plotted versus a daily time course. Light and dark phases and subjective day and night are indicated by white, black, and gray bars, respectively. D, Total daily sleep of $+/+, s r a^{K O} /+$, and $s r a^{K O}$ flies in LD and DD conditions. $\boldsymbol{E}-\boldsymbol{G}$, Waking activity index $(\boldsymbol{E})$, sleep bout number $(\boldsymbol{F})$, and sleep bout length $(\boldsymbol{G})$ in $+/+$ and sra ${ }^{K O}$ mutants. Data in $\boldsymbol{B}$ and $\boldsymbol{D}$ were analyzed by one-way ANOVA followed by Tukey's post hoc analyses. Data in $\boldsymbol{E}-\boldsymbol{G}$ were analyzed by $t$ test. In all cases, NS indicates no significant difference and ${ }^{* * *}$ indicates $p<0.001$. Error bars indicate SEMs.

\section{Materials and Methods}

Fly stocks and maintenance. Flies were reared on conventional corn meal, yeast, glucose, agar medium at $25^{\circ} \mathrm{C}$. All stocks were outcrossed to our wild-type, $w(\mathrm{CS})$ line at least six times to normalize genetic backgrounds (Dura et al., 1993). elav-GAL4 (BL-8765; P\{GAL4-elav.L $\}$ 2) was obtained from the Bloomington Stock Center. sra ${ }^{K O}$ (Takeo et al., 2006), sra ${ }^{G S 3080}$ (Ejima et al., 2004), and UAS-sra (Ejima et al., 2004) lines have been described previously.

Measurement of locomotor activity and sleep. Two- to four-day-old male flies were placed individually in glass tubes (length $6.5 \mathrm{~cm}$; inner diameter, $3 \mathrm{~mm}$ ) containing $\sim 1 \mathrm{~cm}$ of food at one end and a cotton stopper on the other end. Locomotor activity was measured using a Drosophila activity monitor (DAM) (Trikinetics), which records how often flies cross an infrared beam located near the center of each tube. Crossings per minute were recorded over $3 \mathrm{~d}$ in LD conditions followed by $3 \mathrm{~d}$ in DD conditions. Data were processed using a Microsoft Excel-based program described previously (Hendricks et al., 2003). Sleep was defined as 5 or more minutes of continual inactivity and was plotted in $30 \mathrm{~min}$ time bins. A waking activity index was calculated by dividing total daily activity by the length of the active period, which was defined as the total number of minutes where a fly exhibited at least one activity count. Analyses were performed using at least 19 flies per genotype.

Western blot analysis. Two- to four-day-old flies were frozen in liquid $\mathrm{N}_{2}$ and heads were isolated on dry ice. Protein extracts were made by crushing heads in sample loading buffer (50 mm Tris Cl, pH 6.8, $100 \mathrm{~mm}$ DTT, $2 \%$ SDS, $0.1 \%$ bromophenol blue, and $10 \%$ glycerol). Two and a half head equivalents per lane were subjected to $10 \%$ PAGE and transferred to nylon membranes. Blots were probed with rabbit anti-Sra antibody (1:1000) (Takeo et al., 2006) or mouse anti- $\alpha$-tubulin antibody (1:1000) (Sigma-Aldrich) and appropriate HRP-conjugated secondary antibodies (Promega). Chemiluminescent signals were obtained using ECL Western blotting reagents (GE Healthcare) and detected using $\mathrm{x}$-ray film (Bio-rad Laboratories). Densitometric analyses were performed using ImageJ software (http://rsb.info.nih.gov/ij/).

Generation of knock-out lines. CN gene knock-out lines were generated using the ends-out gene targeting technique (Gong and Golic, 2003). Briefly, a knock-out cassette (containing FRTs and I-SceI recognition 
A

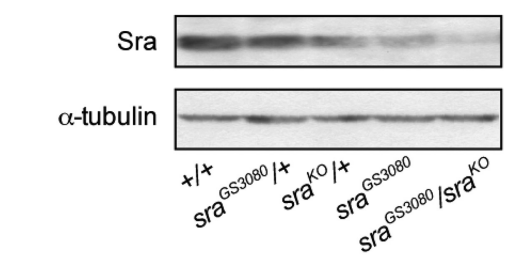

C

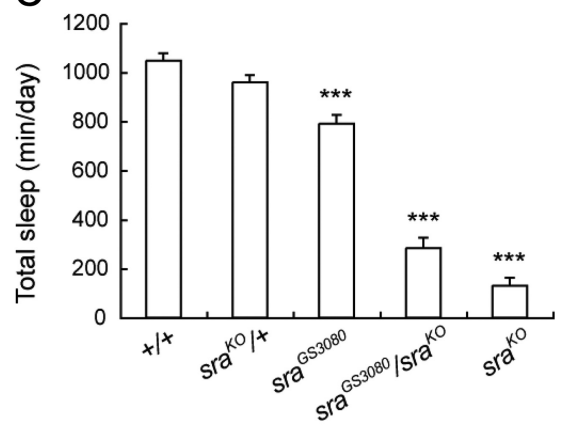

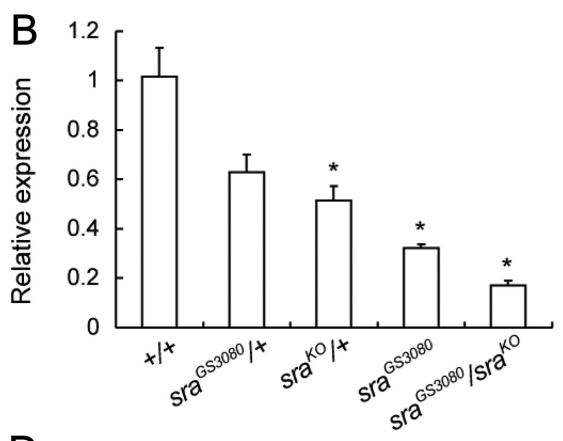

D

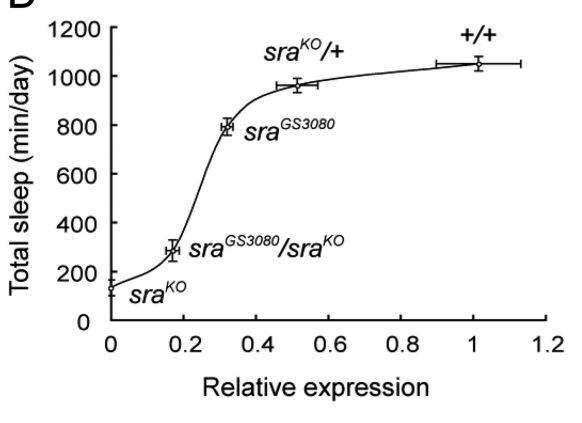

Figure 2. Sleep in sra mutants is correlated with amounts of Sra protein. $\boldsymbol{A}$, A representative Western blot measuring Sra protein amounts in indicated sra mutants. The same blot probed with anti- $\alpha$-tubulin is shown for comparison. $\boldsymbol{B}$, Relative amounts of $S$ ra normalized to $\alpha$-tubulin in sra mutants. One-way ANOVA indicates significant difference in Sra levels due to genotype. ${ }^{*}$ indicates a significant difference $(p<0.05)$ compared to $+/+$, as assayed by Tukey's post hoc analysis. $C$, Total daily sleep plotted for various sra mutants. One-way ANOVA indicates significant differences in sleep due to genotype. ${ }^{* * *}$ indicates significant difference $(p<0.001)$ compared to $+/+$ as assayed by Tukey's post hoc analysis. $D$, Total daily sleep plotted as a function of Sra protein amounts.

sequences surrounding a $5^{\prime}$ homologous region, a $w^{+}$marker, and a $3^{\prime}$ homologous region) was integrated non-homologously into the genome to generate $P\{$ donor\} lines. The knock-out cassette was excised and linearized by crossing the $P\{$ donor $\}$ lines to a [70FLP][70I-SceI] line, which contains heat-inducible FLP recombinase and I-SceI endonuclease transgenes, and heat shocking the progeny 3-5 d after egg laying. Candidate homologous knock-out lines were identified as described previously (Takeo et al., 2006) and confirmed by PCR. P\{donor\} plasmids containing the knock-out cassettes were generated by cloning $\sim 2 \mathrm{~kb}$ of sequence both upstream and downstream of the knock-out locus into the polylinker of $p\{E n d s O u t 2\}$ (Takeo et al., 2006). Primer sequences used for amplifying all upstream and downstream sequences are available upon request. The mini-white gene was inserted in between upstream and downstream sequences in all knock-out constructs, except for the $C a n A 1^{K O}$ construct, which used the hsp::white gene with the GMR enhancer from $p G X$-attP (Huang et al., 2009). Two loxP sites were inserted around the mini-white or white genes to facilitate removal of the marker after identification of knock-out lines. In addition, an attP site was inserted $3^{\prime}$ to the downstream loxP site to allow future integration of constructs into the knock-out site.

Generation of UAS-CanA ${ }^{\text {act }}$ lines. Constitutively active forms of CnA genes were generated as previously described for $P p 2 B-14 D$ (Sullivan and Rubin, 2002). Briefly, CanA $1^{\text {act }}$ was made by inserting a stop codon at amino acid residue 456 (out of 622), $P p 2 B-14 D^{a c t}$ was made by inserting a stop at residue 456 (out of 570) and $C a n A-14 F^{a c t}$ was made by inserting a stop at residue 450 (out of 584). PCR was performed using $3^{\prime}$ primers incorporating a stop codon at the appropriate location and flanking restriction sites. Amplified genes were cloned into a pUASTattB vector (Bischof et al., 2007), which contains an attB site that was used for $\phi \mathrm{C} 31$ dependent integration at the attP site (2L-22A) of $\mathrm{ZH}$-attP-22A flies (Bischof et al., 2007). Transformants were outcrossed to $w(\mathrm{CS})$ flies for six generations to normalize genetic background.

\section{Results}

$s r a^{K O}$ mutants exhibit increased locomotor activity and loss of sleep While studying $\mathrm{Ca}^{2+}$ signaling in egg activation in Drosophila, we noticed that $\mathrm{sra}^{\mathrm{KO}}$ mutants move continuously and do not become quiescent. To characterize this behavioral phenotype more precisely, we measured the activity of these mutants using a Drosophila activity monitor. When wildtype flies are raised in $12 \mathrm{~h}$ light/dark (LD) conditions, they are preferentially active during the times around lights on and lights off (Fig. 1A) (Hendricks et al., 2000; Shaw et al., 2000). After light entrainment, this locomotor behavior continues in continual dark conditions (DD) in a circadian manner (Konopka and Benzer, 1971). In contrast, $s r{ }^{K O}$ flies move throughout the $24 \mathrm{~h}$ day (Fig. 1A) and are approximately twofold more active than wild-type flies in both $\mathrm{LD}$ and DD conditions (Fig. $1 B$ ). Heterozygous $\mathrm{sra}^{\mathrm{KO}} /+$ mutants have identical activity to wild-type flies, indicating that this increased activity is a recessive phenotype.

The continual movement of $s{ }^{K O}$ flies suggested that they may have decreased sleep. From previous work, Drosophila sleep has been defined as 5 or more minutes of continuous inactivity, since these periods are associated with increased arousal threshold, a characteristic of sleep (Hendricks et al., 2000; Shaw et al., 2000). Compared to wild-type flies, $s \mathrm{ra}{ }^{K O}$ flies showed a dramatic loss of sleep, to $\sim 30 \%$ of wild-type amounts (Figs. $1 C, D, 2 C, 3 B$ ). This result was also observed in DD conditions.

To determine whether general hyperactivity could explain the increased daily activity and decreased sleep in $s r{ }^{K O}$ mutants, we calculated locomotor activity, specifically during waking in wildtype and $s{ }^{K O}$ mutants. Waking activity was slightly decreased in LD conditions in $s r a^{K O}$ mutants, while it was only slightly increased in DD conditions (Fig. $1 E$ ). Thus the increase in daily activity of $\mathrm{sra}^{\mathrm{KO}}$ mutants is due to increased time spent in a waking state, rather than to increased activity during waking.

Total sleep amounts depend upon both the frequency of sleep bouts and the length of time of each sleep bout. The number of daily sleep bouts was the same in $s r a^{K O}$ mutants and wild-type flies, in both $\mathrm{LD}$ and DD conditions (Fig. $1 F$ ). In contrast, the average sleep bout length in $s r{ }^{K O}$ mutants was decreased to $\sim 30 \%$ that of wild type, in $\mathrm{LD}$ and $\mathrm{DD}$ conditions (Fig. $1 G$ ), indicating that decreased sleep in $\mathrm{sra}^{K O}$ was due to a decrease in sleep maintenance, rather than to a decrease in sleep onset.

\section{Sleep is correlated with levels of Sra protein}

To verify that the $s r{ }^{K O}$ sleep phenotype is due to mutation of the sra locus, we measured sleep in a second mutant, $s r a^{G S 3080}$, which has a $P$-element transposon insertion in the intron of the sra gene (Ejima et al., 2004). This insertion reduces Sra protein levels to $30 \%$ of wild-type levels (Fig. $2 A, B$ ). Surprisingly, sleep was only mildly reduced in $s \mathrm{ra}^{\mathrm{GS} 3080}$ mutants (Fig. $2 C$ ), suggesting that relatively low amounts of Sra are sufficient for normal sleep. We tested this idea by measuring Sra protein levels (Fig. $2 B$ ) and daily amounts of sleep (Fig. 2C) in several sra homozygous, heterozy- 


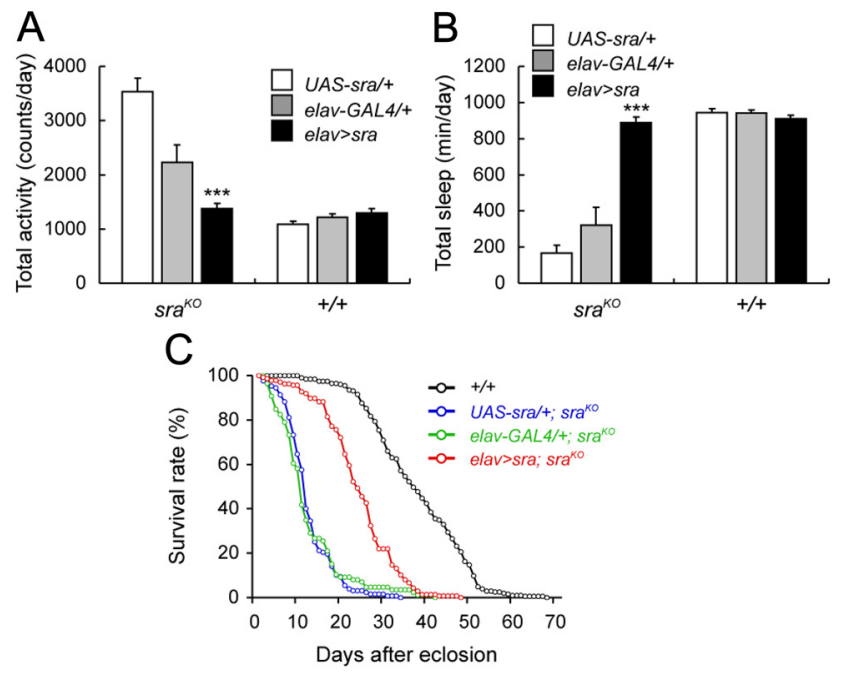

Figure 3. Expression of sra in neurons rescues activity, sleep and lifespan phenotypes of $s r a^{K O}$ mutants. $A$, Daily activity of UAS-sra/ +; $s r a^{K O}$ and elav-GAL4/+; $s r a^{K O}$ flies is significantly higher than wild type, while activity in elav $>s r a ; s r a{ }^{K O}$ flies is reduced back to wild-type levels. Neuronal expression of sra in a wild-type background has no effect on activity. $\boldsymbol{B}$, Neuronal expression of sra rescues $s r{ }^{K O}$ sleep defects (elav $>$ sra; $s r{ }^{K O}$ ), while it has no effects in a wild-type background. Driver alone and UAS-sra alone controls do not rescue $\mathrm{sra}^{K O}$ sleep defects. In $\boldsymbol{A}$ and $\boldsymbol{B},{ }^{* * *}$ indicates significant differences between elav $>$ sra and both UAS-sra alone and elav-GAL4 alone controls in the $\mathrm{Sra}^{\mathrm{KO}}$ background $(p<0.001)$. No significant differences were detected in the $+/+$ background. C, Survival curves for male wild-type $(+/+)$, elav $>$ sra; $s r a^{K O}$, elav-GAL4/+; sra ${ }^{K O}$, and UAS-sra/+; $s r a^{K O}$ flies. Neuronal expression of sra partially rescues the reduced lifespan of $\mathrm{Sra}^{\mathrm{KO}}$ mutants. Wild-type males have an average lifespan of $37.82 \pm 0.76 \mathrm{~d}$, while elav $>$ sra; $s r^{K O}$, elav-GAL4/+; $\mathrm{sra}^{K O}$, and UAS-sra $/+; \mathrm{sra}^{K O}$ flies have average lifespans of $24.30 \pm 0.70,12.60 \pm 0.83$, and $12.65 \pm 0.48 \mathrm{~d}$, respectively.

gous, and transheterozygous mutants. As seen in Figure $2 D$, reducing Sra to $50 \%$ of wild-type levels does not significantly affect sleep. Decreasing Sra further, to $30 \%$ of wild-type levels, has a mild effect, while a further reduction to $20 \%$ results in a precipitous decrease in time spent asleep. Thus, relatively large reductions in Sra function are necessary to affect sleep, and decreased sleep is highly correlated with reduced levels of Sra protein.

\section{Neuronal expression of sra rescues $s r a^{K O}$ phenotypes}

If increased locomotor activity and decreased sleep in mutants are due to reduced amounts of Sra, these phenotypes should be rescued by expression of wild-type sra. To test this, we expressed a UAS-sra ${ }^{+}$transgene pan-neuronally using an elav-GAL4 driver in a $s r a^{K O}$ background. While control $s r a^{K O}$ lines containing UAS-sra ${ }^{+}$alone or elav-GAL4 alone had over twice the locomotor activity of wild-type flies (compare Fig. $3 A$ with Fig. $1 B$ ), activity was reduced back to the wild-type levels in elav-GAL4/ $U A S-s r a^{+} ; s r a^{K O}$ flies (elav>sra; sra ${ }^{K O}$ ). Similarly, sleep was completely restored to wild-type amounts in this genotype (Fig. $3 B$ ). Overexpression of $\mathrm{sra}^{+}$in a wild-type background did not affect activity or sleep, demonstrating that reduced activity and increased sleep is due to rescue, rather than to a nonspecific effect of $\mathrm{sra}^{+}$overexpression.

\section{$s r a^{K O}$ mutants have a significantly reduced lifespan}

We observed that $\mathrm{sra}^{K O}$ flies tend to die much earlier than wildtype flies. Sleep is thought to be an essential biological function, since sleep deprivation can lead to death (Rechtschaffen et al., 1983; Shaw et al., 2002). In addition, several previously identified sleep mutants have decreased lifespans, suggesting that sleep and organismal lifespan may be linked (Shaw et al., 2002; Cirelli et al.,
2005; Seugnet et al., 2009) (but also see Kume et al., 2005). Thus we measured the lifespan of $\mathrm{sra}^{K O}$ mutants and determined that it was significantly reduced compared to wild type. While wild-type flies have mean ( \pm SEM) lifespans of $37.82 \pm 0.76 \mathrm{~d}$ for males and $37.36 \pm 0.76 \mathrm{~d}$ for females, $s r a^{K O}$ mutants (elav-GAL4/+; sra ${ }^{K O}$ ) live an average of only $12.60 \pm 0.83 \mathrm{~d}$ for males (Fig. $3 C$ ), and $12.81 \pm 0.48 \mathrm{~d}$ for females (data not shown). This shortened lifespan can be partially rescued by expressing sra in neurons $\left(\right.$ elav $\left.>s r a ; s r a^{K O}\right)$, indicating that, similar to sleep, lifespan is dependent on neuronal Sra function.

\section{Calcineurin regulates sleep and locomotor activity}

Why do sra mutants sleep less than wild-type flies? Sra's role as a $\mathrm{CN}$ regulator (Horner et al., 2006; Takeo et al., 2006) suggested that $\mathrm{CN}$ may play a role in regulating sleep. To test this possibility, we generated individual knock-outs of all Drosophila CN genes and measured sleep and locomotion. In Drosophila, three genes, CanA-14F, Pp2B-14D, and CanA1, encode catalytic subunits, and two genes, $C a n B$ and $C a n B 2$, encode regulatory subunits. We generated knock-outs of all of these genes by homologous recombination (Fig. 4). As seen in Figure $5 \mathrm{~A}$, all knock-out lines affected total daily locomotor activity except for $C a n B 2^{K O}$. In addition, all lines significantly affected daily amounts of sleep (Fig. 5D). However, the extent to which individual $\mathrm{CN}$ gene knock-outs affected locomotion and sleep varied widely. Among the regulatory subunits, $C a n B 2^{K O}$ had the smallest effect on these behaviors (a 10\% decrease in the amount of sleep). In contrast, knock-out of the other regulatory subunit, $C a n B$, dramatically increased locomotion and decreased the amount of sleep (Fig. $5 A, C, D, F$ ) (an $\sim 50 \%$ decrease in the amount of sleep). These phenotypes were similar to those of $s \mathrm{sa}^{K O}$ flies. Interestingly, while $\operatorname{CanB}^{K O} \mathrm{mu}$ tants had decreased sleep amounts to $\sim 50 \%$ of wild-type in both daytime and nighttime in LD conditions, in DD conditions, these mutants showed a further decrease in sleep during the subjective day to $20 \%$ of wild type. While we are not certain why this occurs, this result suggests that decreasing $\mathrm{CN}$ activity enhances sleep in the presence of light (Fig. $5 F$ ).

Among the catalytic subunit mutants, $P p 2 B-14 D^{K O}$ flies had a small but significant $25 \%$ increase in locomotion and a $10 \%$ decrease in the amount of sleep. CanA $1^{K O}$ flies had an opposite effect of slightly decreasing total activity and slightly increasing the amount of sleep. However, we believe this apparent sleep effect is artifactual, due to reduced mobility of $\operatorname{CanA} 1^{K O}$ flies. CanA$14 F^{K O}$ flies showed the largest effects on activity and sleep, increasing locomotor activity approximately threefold and decreasing the amount of sleep over twofold (Fig. $5 A, B, D, E$ ). Similar to $C_{a n} B^{K O}$ mutants, $C a n A-14 F^{K O}$ flies also sleep less during the subjective day in DD conditions than they do in LD conditions (Fig. 5E), again suggesting that while $\mathrm{CN}$ plays an important role in sleep in general, it may also have a second, more specific role in suppressing sleep in the presence of light.

Our results indicate that all $\mathrm{CN}$ subunits, with the possible exception of CanA1, contribute to daily locomotion and sleep. However, they also indicate that the functions of $\mathrm{CN}$ subunits are not identical, and suggest that the CanA-14F catalytic and the CanB regulatory subunits are the most important in controlling sleep amounts.

\section{Constitutively active calcineurin inhibits sleep}

If decreasing $\mathrm{CN}$ reduces sleep, we reasoned that increasing $\mathrm{CN}$ activity might increase sleep. $\mathrm{Ca}^{2+} / \mathrm{CaM}$-independent, constitutively active $\mathrm{CN}$ can be made by truncating the $\mathrm{C}$-terminal $\mathrm{Ca}^{2+}$ / $\mathrm{CaM}$ binding regions and autoinhibitory domains of $\mathrm{CnA}$ 
A Calcineurin A subunit genes

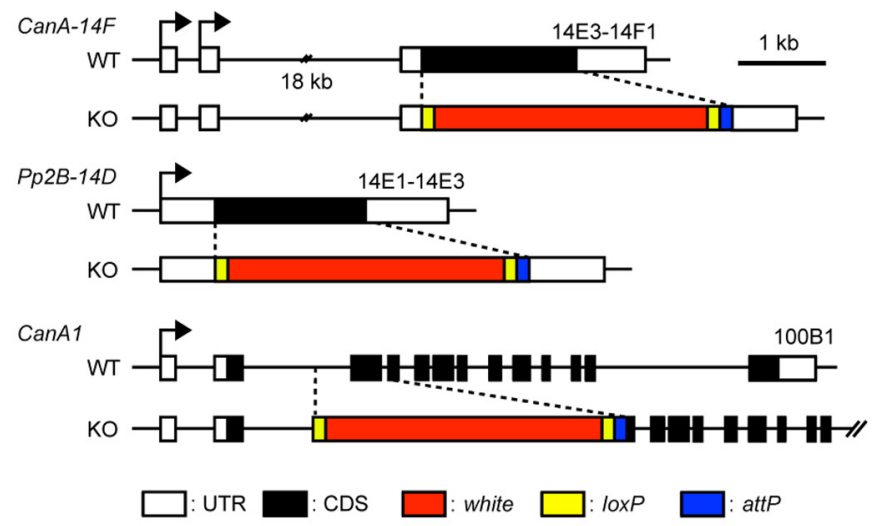

B Verification of targeting events
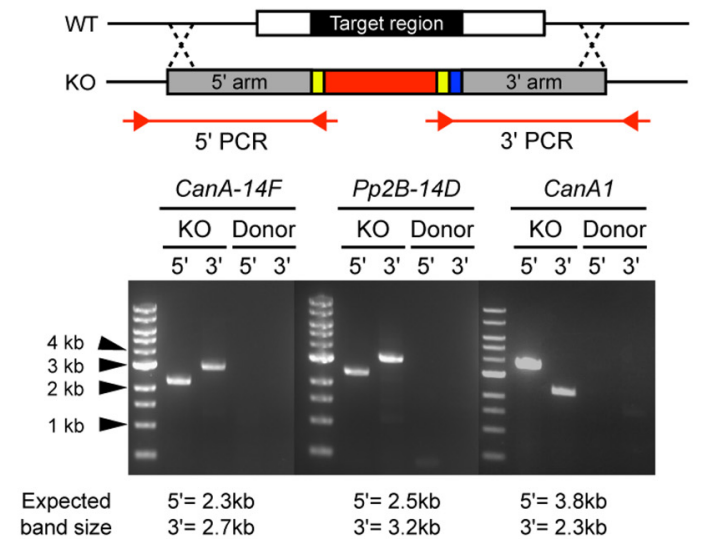

Figure 4. Generation of $\mathrm{CN}$ gene knock-out mutants. $A$, Schematic diagram of genomic loci for wild-type and knock-out alleles of CanA-14F, $P p 2 B-14 D$, and CanA1. CanA-14 $F^{K O}$ and $P p 2 B-14 D^{K O}$ constructs precisely remove all of the protein coding regions of the corresponding genes, while the $C a n A 7^{K O}$ construct removes the second and third exons. $B$, Verification of knock-outs. Knockout lines were confirmed by PCR, shown schematically in the upper panel. Two separate PCRs were performed per knock-out candidate, one corresponding to the $5^{\prime}$ end of the knock-out locus and the second corresponding to the $3^{\prime}$ end. Internal primers were made bridging the loxP and white regions, while external primers were made to areas outside the homologous region. Knock-out lines show bands of the expected size (KO), while $P\{d o n o r\}$ lines, where the knock-out cassette is integrated at a

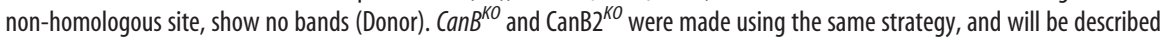
elsewhere.

catalytic subunits (O'Keefe et al., 1992). Thus, we constructed $U A S-$ Can $^{\text {act }}$ transgenic lines, which express constitutively active versions of each of the CanA catalytic subunit genes under GAL4 control. We crossed our lines to an elav-GAL4 line and measured sleep in the progeny. Neuronal expression of all three CanA ${ }^{\text {act }}$ transgenes caused significant increases in activity and decreases in sleep, indicating that both increases and decreases in $\mathrm{CN}$ activity increase locomotor activity and decrease sleep (Fig. 5G).

\section{Genetic interaction between sra and calcineurin}

Mutations in both sra and CN genes increase locomotion and decrease amounts of sleep. Are these two phenotypes linked or independent? To answer this question, we made double mutants containing CanA $-14 F^{K O}$ or $P p 2 B-14 D^{K O}$ and sra hypomorphic mutations, to see whether effects on sleep were purely additive or whether we could see genetic interactions between these mutations. In this experiment, we used $\mathrm{sra}^{G S 3080} / \mathrm{sra}^{K O}$ transheterozygotes, which sleep approximately one-third of the amount that wild-type flies sleep (Fig. 2C), allowing for easy identification of both increases and decreases in sleep. While $\mathrm{sra}^{G S 3080} / \mathrm{sra}^{K O}$ flies have reduced sleep, both CanA-14F $F^{K O}$ (Fig. 5G) and $P p 2 B-14 D^{K O}$ (Fig. 5H) mutations partially suppressed this phenotype. In contrast, single $\operatorname{CanA}-14 F^{K O}$ or $P p 2 B-14 D^{K O}$ mutations decreased amounts of sleep. These results indicate that sleep phenotypes in sra and $\mathrm{CN}$ gene mutants occur through a common genetic pathway, and that Sra antagonizes $\mathrm{CN}$ under our experimental conditions. Increased $\mathrm{CN}$ activity in sra hypomorphic mutants leads to a decrease in the amount of sleep, which can be suppressed by deleting one of the CN catalytic subunits.

\section{Discussion}

$\mathrm{Ca}^{2+} / \mathrm{CaM}$ signaling has been implicated in many different biological processes, but a definite role of $\mathrm{Ca}^{2+} / \mathrm{CaM}$ signaling in sleep has not previously been shown. Here we demonstrate that mutations in $\mathrm{CN}$ genes, $C a n A-14 F$ and $C a n B$, and in the $C N$ regulator, sra, strongly decrease amounts of sleep and increase total daily activity. Sleep in $\mathrm{sra}^{K O}$ mutants is reduced to almost nonexistent levels.

Deletions of all individual Drosophila $\mathrm{CN}$ genes have at least minor effects on sleep. However, knock-out of one catalytic subunit gene, $C a n A-14 F$, and of one regulatory subunit gene, $C a n B$, have particularly pronounced effects, indicating that these are the predominant subunits regulating sleep. It is possible that sleep is regulated strictly by these subunits and that the smaller effects produced by deleting the other subunits occurs indirectly, due to compensatory changes in $\operatorname{CanA}$ $14 F$ and $C a n B$. On the other hand, $s r a^{K O}$ mutants show more severe sleep effects than any of the single $\mathrm{CN}$ gene mutants, suggesting that several $\mathrm{CN}$ isoforms affect sleep directly to different extents, and that the $\mathrm{sra}^{K O}$ phenotypes reflect additive effects of individual $\mathrm{CN}$ gene knock-outs.

Although Sra and other RCANs are well known as $\mathrm{CN}$ regulators (Hilioti and Cunningham, 2003; Davies et al., 2007; Stie and Fox, 2008), it is uncertain whether RCANs function to activate or inhibit CN activity (Kingsbury and Cunningham, 2000; Vega et al., 2003; Takeo et al., 2010). In our assays, both increases and decreases in $\mathrm{CN}$ activity inhibit sleep, suggesting that Sra may affect sleep through either mechanism. However, knock-outs of CanA-14F or Pp2B-14D suppress hypomorphic sra sleep phenotypes, supporting a model where decreased amount of sleep in sra mutants is caused by increased CN activity. While this result is clear, recent work has demonstrated that Sra is phosphorylated at two different serine residues, and that phosphorylation at these sites determines whether Sra activates or represses $\mathrm{CN}$ activity (Vega et al., 2002; Hilioti et al., 2004; Abbasi et al., 2005; Mehta et al., 2009). Thus, although the molecular mechanisms controlling Sra regulation of $\mathrm{CN}$ need to be further clarified, our data suggest that Sra functions to inhibit $\mathrm{CN}$ activity, at least under the conditions we examined.

Increases in sleep have been associated with memory formation (Ganguly-Fitzgerald et al., 2006), while sleep deprivation has 
A
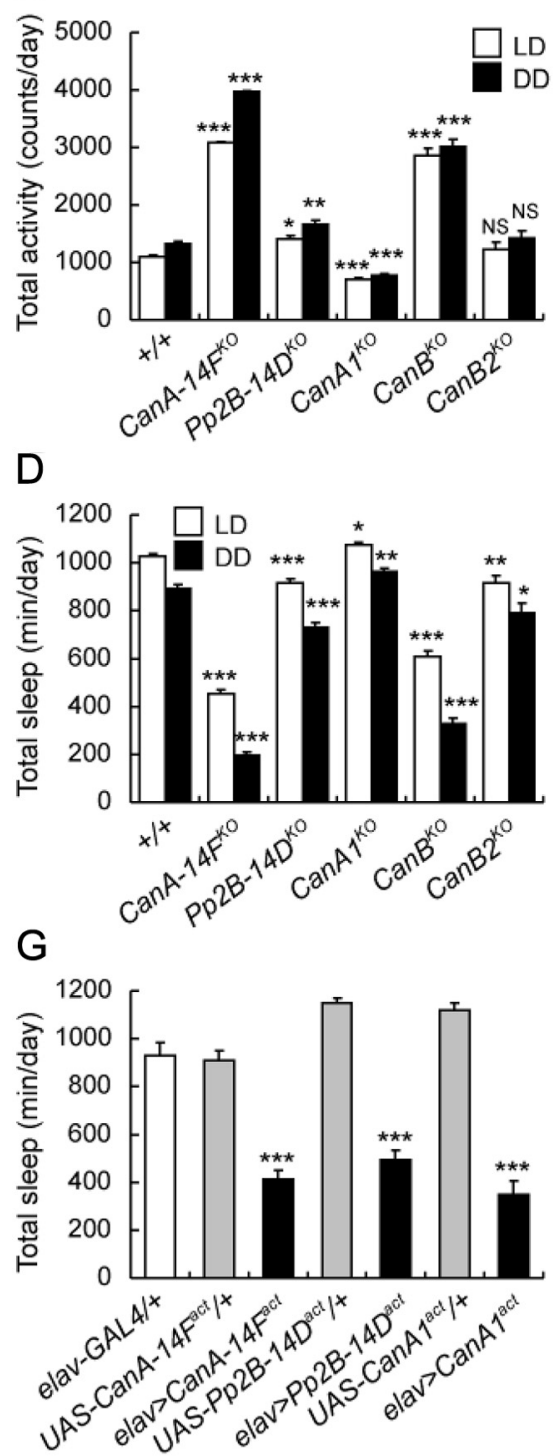

B

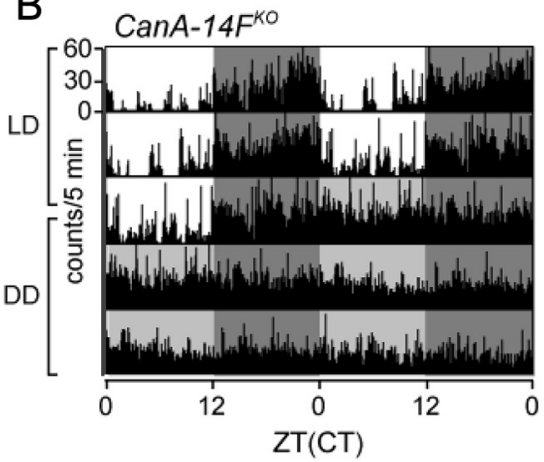

$\mathrm{E}$

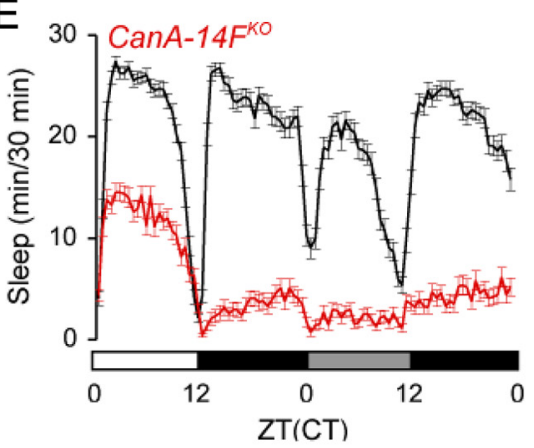

$\mathrm{H}$

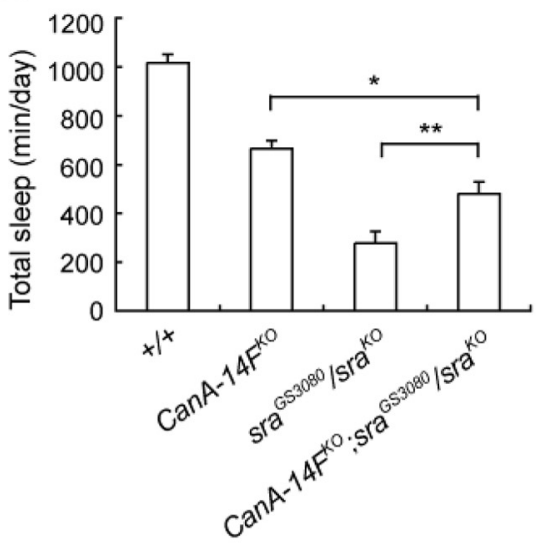

C

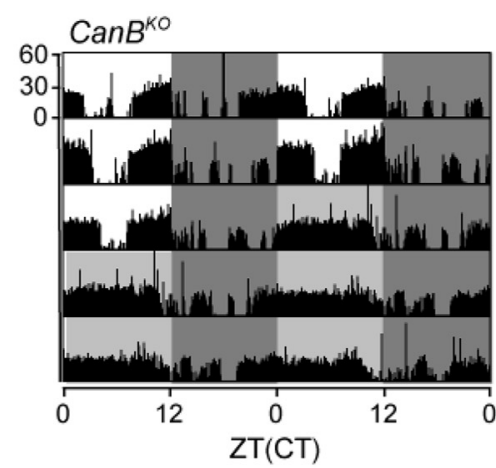

F

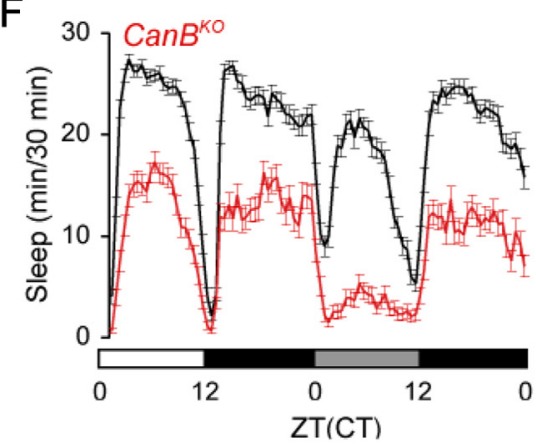

I

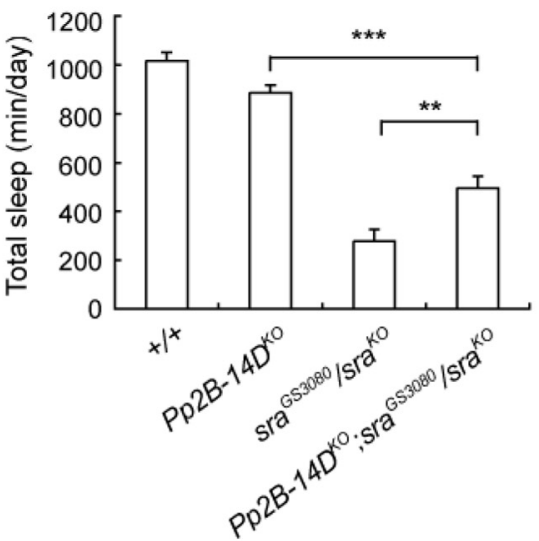

Figure 5. Both decreases and increases in $C N$ activity inhibit sleep. $\boldsymbol{A}, \boldsymbol{D}$, Total daily activity $(\boldsymbol{A})$ and total daily sleep $(\boldsymbol{D})$ in knock-out mutants of all $C \mathrm{CN}$ catalytic and regulatory subunit genes. Two-way ANOVA indicates significant differences in activity and sleep due to genotype, LD/DD cycle, and interaction between genotype and LD/DD cycle. All mutants, except CanB2 ${ }^{K O}$, show significant effects on activity, and all mutants show significant effects on sleep. $B, C, E, F$, Knock-outs of the CanA-14F catalytic subunit gene and of the CanB regulatory subunit gene show the most dramatic effects on activity and sleep, with approximately threefold increases in activity $(\boldsymbol{B}, \boldsymbol{C})$ and twofold decreases in sleep $(\boldsymbol{E}, \boldsymbol{F})$. $\boldsymbol{G}$, Neuronal expression of all three CanA ${ }^{a c t}$ transgenes significantly reduces total daily sleep. One-way ANOVA indicates significant differences in sleep due to genotype. Bonferroni post hoc analyses indicate significant differences in sleep between wild-type and all three elav $>$ CanA $A^{a c t}$ lines $\left(p<0.001\right.$ in all cases), but no differences between wild-type and elav-GAL4/+ and all CanA $A^{a c t} /+$ controls. $\boldsymbol{H}, \boldsymbol{I}$, Genetic interaction between hypomorphic sra mutants $\left(\mathrm{sra} \mathrm{G}^{63080} / \mathrm{sra} \mathrm{C}^{K O}\right.$ ) and $\operatorname{CanA}-14 \mathrm{~F}^{K O}(\boldsymbol{H})$ or $P$ P $2 B-14 \mathrm{D}^{K O}(\mathrm{I})$. Total daily sleep amounts of wild-type, double mutants, and corresponding single mutant controls are shown. Both CanA-14KKO and $P p 2 B-14 D^{K O}$ suppress sra sleep phenotypes. In both $\boldsymbol{H}$ and $\boldsymbol{I}$, one-way ANOVA indicates significant differences due to genotype. Bonferroni post hoc analyses indicate that amounts of sleep in both double mutants are significantly different from that of corresponding single mutants. Data in bar graphs and sleep profiles represent means \pm SEM; ${ }^{* * *}$, ${ }^{* *}$, and ${ }^{*}$ indicate statistically significant differences with $p<0.001, p<0.01$, and $p<0.05$, respectively. NS indicates that differences are not statistically significant.

been associated with inhibition of learning and memory (Seugnet et al., 2008; Li et al., 2009; Vecsey et al., 2009). Thus sleep is thought to be important for strengthening or maintaining synapses coding for memories, and decreases in sleep amounts may correlate with memory impairment. Conversely, the need to sleep may decrease when memories are not formed. Most, if not all, sleep mutants have memory defects, and $\mathrm{CN}$ and sra have also been shown to be important for memory formation and synaptic function in several species (Zeng et al., 2001; Chang et al., 2003; Lee and Ahnn, 2004; Baumgärtel et al., 2008; Chang and Min, 2009; Christie-Fougere et al., 2009). Furthermore, both increases and decreases in $\mathrm{CN}$ and Sra activity are associated with decreased memory (Mansuy et al., 1998; Foster et al., 2001; Chang et al., 2003). These results support a model where sleep and memory are linked; sleep need in $\mathrm{CN}$ and sra mutants may be decreased because memory and new memory-dependent synaptic connections are not made.

Our data are consistent with several possible roles of Sra and $\mathrm{CN}$ in sleep regulation. Sra and $\mathrm{CN}$ may be involved during development for the formation of neuronal circuits necessary for normal sleep, they may be involved acutely at the adult stage to directly regulate sleep, or both. Currently, all conditional expres- 
sion systems that we have tried, including rescuing sra conditionally using available sra transgenes under heat-shock (Takeo et al., 2006) and gene switch control (Osterwalder et al., 2001) and inhibiting sra conditionally using RNAi transgenes, have been toxic to sra mutants, rescued sra in the absence of inducer, or failed to inhibit sra enough to produce sleep phenotypes. We believe that the extremely low amount of sra required for sleep (20-30\% of wild-type amounts) is responsible for most of these problems.

Altered $\mathrm{CN}$ activity has been associated with several neurological disorders. For example, Down's syndrome critical region 1 (DSCR1), a homolog of the sra gene, was identified from extensive studies of Down's syndrome (DS) patients with partial trisomy 21 (Epstein, 1995). Increased expression of sra is thought to alter $\mathrm{CN}$ activity, contributing to the mental defects observed in DS patients. In addition, a locus associated with susceptibility to schizophrenia has been mapped to PPP3CC, which encodes the mammalian CN gamma catalytic subunit (Gerber et al., 2003). Furthermore, mice with forebrain-specific knock-outs of CN display a spectrum of disorders strikingly similar to behaviors observed in schizophrenia patients (Miyakawa et al., 2003). Although alterations in sleep have been associated with both DS and schizophrenia, mechanistic connections between these diseases and sleep have been lacking. Our data suggest that these diseases may affect sleep through altered CN activity. It will be of great interest to determine whether both mental defects and sleep defects are caused by altered $\mathrm{Ca}^{2+} / \mathrm{CaM}$-dependent signaling in similar neuronal pathways.

\section{References}

Abbasi S, Su B, Kellems RE, Yang J, Xia Y (2005) The essential role of MEKK3 signaling in angiotensin II-induced calcineurin/nuclear factor of activated T-cells activation. J Biol Chem 280:36737-36746.

Agosto J, Choi JC, Parisky KM, Stilwell G, Rosbash M, Griffith LC (2008) Modulation of GABAA receptor desensitization uncouples sleep onset and maintenance in Drosophila. Nat Neurosci 11:354-359.

Baumgärtel K, Genoux D, Welzl H, Tweedie-Cullen RY, Koshibu K, Livingstone-Zatchej M, Mamie C, Mansuy IM (2008) Control of the establishment of aversive memory by calcineurin and Zif268. Nat Neurosci 11:572-578.

Bischof J, Maeda RK, Hediger M, Karch F, Basler K (2007) An optimized transgenesis system for Drosophila using germ-line-specific phiC31 integrases. Proc Natl Acad Sci U S A 104:3312-3317.

Chang KT, Min KT (2009) Upregulation of three Drosophila homologs of human chromosome 21 genes alters synaptic function: implications for Down syndrome. Proc Natl Acad Sci U S A 106:17117-17122.

Chang KT, Shi YJ, Min KT (2003) The Drosophila homolog of Down's syndrome critical region 1 gene regulates learning: implications for mental retardation. Proc Natl Acad Sci U S A 100:15794-15799.

Christie-Fougere MM, Darby-King A, Harley CW, McLean JH (2009) Calcineurin inhibition eliminates the normal inverted $U$ curve, enhances acquisition and prolongs memory in a mammalian $3^{\prime}-5^{\prime}$-cyclic AMPdependent learning paradigm. Neuroscience 158:1277-1283.

Chung BY, Kilman VL, Keath JR, Pitman JL, Allada R (2009) The GABA(A) receptor RDL acts in peptidergic PDF neurons to promote sleep in Drosophila. Curr Biol 19:386-390.

Cirelli C, Bushey D, Hill S, Huber R, Kreber R, Ganetzky B, Tononi G (2005) Reduced sleep in Drosophila Shaker mutants. Nature 434:1087-1092.

Crocker A, Sehgal A (2008) Octopamine regulates sleep in Drosophila through protein kinase A-dependent mechanisms. J Neurosci 28:9377-9385.

Davies KJ, Ermak G, Rothermel BA, Pritchard M, Heitman J, Ahnn J, Henrique-Silva F, Crawford D, Canaider S, Strippoli P, Carinci P, Min KT, Fox DS, Cunningham KW, Bassel-Duby R, Olson EN, Zhang Z, Williams RS, Gerber HP, Pérez-Riba M, et al. (2007) Renaming the DSCR1/ Adapt78 gene family as RCAN: regulators of calcineurin. FASEB J 21:3023-3028.

Dura JM, Preat T, Tully T (1993) Identification of linotte, a new gene affect- ing learning and memory in Drosophila melanogaster. J Neurogenet 9:1-14.

Ejima A, Tsuda M, Takeo S, Ishii K, Matsuo T, Aigaki T (2004) Expression level of sarah, a homolog of DSCR1, is critical for ovulation and female courtship behavior in Drosophila melanogaster. Genetics 168:2077-2087.

Epstein CJ (1995) Down syndrome (trisomy 21). In: The metabolic and molecular bases of inherited disease (Scriver CR, Beaudet AL, Sly WS, Vaile D, eds), pp 749-794. New York: McGraw-Hill.

Foster TC, Sharrow KM, Masse JR, Norris CM, Kumar A (2001) Calcineurin links $\mathrm{Ca}^{2+}$ dysregulation with brain aging. J Neurosci 21:4066-4073.

Ganguly-Fitzgerald I, Donlea J, Shaw PJ (2006) Waking experience affects sleep need in Drosophila. Science 313:1775-1781.

Gerber DJ, Hall D, Miyakawa T, Demars S, Gogos JA, Karayiorgou M, Tonegawa S (2003) Evidence for association of schizophrenia with genetic variation in the $8 \mathrm{p} 21.3$ gene, PPP3CC, encoding the calcineurin gamma subunit. Proc Natl Acad Sci U S A 100:8993-8998.

Gong WJ, Golic KG (2003) Ends-out, or replacement, gene targeting in Drosophila. Proc Natl Acad Sci U S A 100:2556-2561.

Görlach J, Fox DS, Cutler NS, Cox GM, Perfect JR, Heitman J (2000) Identification and characterization of a highly conserved calcineurin binding protein, CBP1/calcipressin, in Cryptococcus neoformans. EMBO J 19:3618-3629.

Hendricks JC, Finn SM, Panckeri KA, Chavkin J, Williams JA, Sehgal A, Pack AI (2000) Rest in Drosophila is a sleep-like state. Neuron 25:129-138.

Hendricks JC, Lu S, Kume K, Yin JC, Yang Z, Sehgal A (2003) Gender dimorphism in the role of cycle (BMAL1) in rest, rest regulation, and longevity in Drosophila melanogaster. J Biol Rhythms 18:12-25.

Hilioti Z, Cunningham KW (2003) The RCN family of calcineurin regulators. Biochem Biophys Res Commun 311:1089-1093.

Hilioti Z, Gallagher DA, Low-Nam ST, Ramaswamy P, Gajer P, Kingsbury TJ, Birchwood CJ, Levchenko A, Cunningham KW (2004) GSK-3 kinases enhance calcineurin signaling by phosphorylation of RCNs. Genes Dev 18:35-47.

Horner VL, Czank A, Jang JK, Singh N, Williams BC, Puro J, Kubli E, Hanes SD, McKim KS, Wolfner MF, Goldberg ML (2006) The Drosophila calcipressin sarah is required for several aspects of egg activation. Curr Biol 16:1441-1446.

Huang J, Zhou W, Dong W, Watson AM, Hong Y (2009) From the cover: directed, efficient, and versatile modifications of the Drosophila genome by genomic engineering. Proc Natl Acad Sci U S A 106:8284-8289.

Kingsbury TJ, Cunningham KW (2000) A conserved family of calcineurin regulators. Genes Dev 14:1595-1604

Konopka RJ, Benzer S (1971) Clock mutants of Drosophila melanogaster. Proc Natl Acad Sci U S A 68:2112-2116.

Kume K, Kume S, Park SK, Hirsh J, Jackson FR (2005) Dopamine is a regulator of arousal in the fruit fly. J Neurosci 25:7377-7384.

Lee JI, Ahnn J (2004) Calcineurin in animal behavior. Mol Cells 17:390-396.

Li X, Yu F, Guo A (2009) Sleep deprivation specifically impairs short-term olfactory memory in Drosophila. Sleep 32:1417-1424.

Mansuy IM, Mayford M, Jacob B, Kandel ER, Bach ME (1998) Restricted and regulated overexpression reveals calcineurin as a key component in the transition from short-term to long-term memory. Cell 92:39-49.

Mehta S, Li H, Hogan PG, Cunningham KW (2009) Domain architecture of the regulators of calcineurin (RCANs) and identification of a divergent RCAN in yeast. Mol Cell Biol 29:2777-2793.

Miyakawa T, Leiter LM, Gerber DJ, Gainetdinov RR, Sotnikova TD, Zeng H, Caron MG, Tonegawa S (2003) Conditional calcineurin knockout mice exhibit multiple abnormal behaviors related to schizophrenia. Proc Natl Acad Sci U S A 100:8987-8992.

O’Keefe SJ, Tamura J, Kincaid RL, Tocci MJ, O’Neill EA (1992) FK-506- and CsA-sensitive activation of the interleukin-2 promoter by calcineurin. Nature 357:692-694.

Osterwalder T, Yoon KS, White BH, Keshishian H (2001) A conditional tissue-specific transgene expression system using inducible GAL4. Proc Natl Acad Sci U S A 98:12596-12601.

Parisky KM, Agosto J, Pulver SR, Shang Y, Kuklin E, Hodge JJ, Kang K, Liu X, Garrity PA, Rosbash M, Griffith LC (2008) PDF cells are a GABAresponsive wake-promoting component of the Drosophila sleep circuit. Neuron 60:672-682.

Rechtschaffen A, Gilliland MA, Bergmann BM, Winter JB (1983) Physio- 
logical correlates of prolonged sleep deprivation in rats. Science 221:182-184.

Rusnak F, Mertz P (2000) Calcineurin: form and function. Physiol Rev 80:1483-1521.

Seugnet L, Suzuki Y, Vine L, Gottschalk L, Shaw PJ (2008) D1 receptor activation in the mushroom bodies rescues sleep-loss-induced learning impairments in Drosophila. Curr Biol 18:1110-1117.

Seugnet L, Suzuki Y, Thimgan M, Donlea J, Gimbel SI, Gottschalk L, Duntley SP, Shaw PJ (2009) Identifying sleep regulatory genes using a Drosophila model of insomnia. J Neurosci 29:7148-7157.

Shaw PJ, Cirelli C, Greenspan RJ, Tononi G (2000) Correlates of sleep and waking in Drosophila melanogaster. Science 287:1834-1837.

Shaw PJ, Tononi G, Greenspan RJ, Robinson DF (2002) Stress response genes protect against lethal effects of sleep deprivation in Drosophila. Nature 417:287-291.

Stie J, Fox D (2008) Calcineurin regulation in fungi and beyond. Eukaryot Cell 7:177-186.

Sullivan KM, Rubin GM (2002) The Ca(2+)-calmodulin-activated protein phosphatase calcineurin negatively regulates EGF receptor signaling in Drosophila development. Genetics 161:183-193.

Takeo S, Tsuda M, Akahori S, Matsuo T, Aigaki T (2006) The calcineurin regulator sra plays an essential role in female meiosis in Drosophila. Curr Biol 16:1435-1440.

Takeo S, Hawley RS, Aigaki T (2010) Calcineurin and its regulation by Sra/ RCAN is required for completion of meiosis in Drosophila. Dev Biol 344:957-967.

Vecsey CG, Baillie GS, Jaganath D, Havekes R, Daniels A, Wimmer M, Huang T, Brown KM, Li XY, Descalzi G, Kim SS, Chen T, Shang YZ, Zhuo M, Houslay MD, Abel T (2009) Sleep deprivation impairs cAMP signalling in the hippocampus. Nature 461:1122-1125.

Vega RB, Yang J, Rothermel BA, Bassel-Duby R, Williams RS (2002) Multiple domains of MCIP1 contribute to inhibition of calcineurin activity. J Biol Chem 277:30401-30407.

Vega RB, Rothermel BA, Weinheimer CJ, Kovacs A, Naseem RH, BasselDuby R, Williams RS, Olson EN (2003) Dual roles of modulatory calcineurin-interacting protein 1 in cardiac hypertrophy. Proc Natl Acad Sci U S A 100:669-674.

Yuan Q, Joiner WJ, Sehgal A (2006) A sleep-promoting role for the Drosophila serotonin receptor 1A. Curr Biol 16:1051-1062.

Zeng H, Chattarji S, Barbarosie M, Rondi-Reig L, Philpot BD, Miyakawa T, Bear MF, Tonegawa S (2001) Forebrain-specific calcineurin knockout selectively impairs bidirectional synaptic plasticity and working/episodiclike memory. Cell 107:617-629. 